\title{
Multiplexing Capabilities of Biosensors for Clinical Diagnostics
}

\author{
Johnson K-K Ng and Samuel S Chong \\ National University of Singapore \\ Singapore
}

\section{Introduction}

The detection of biomolecules, be it proteins or nucleic acids such as DNA or RNA, is a critical process in biomedical research and clinical diagnostics. With the former, it helps us to unravel the complexity of our human body, and provides important information down at the cellular and sub-cellular level that allows us to better understand what our bodies are comprised off, how they function, how they respond to disease and aging, or why they fail to respond. This information, when applied to clinical diagnostics, help better manage our health and enhance the quality of life.

To generate any meaningful or conclusive information for clinical diagnostics, it is often needed to detect several targets simultaneously. Therefore technologies for performing biomolecular detection must be able to interrogate several targets at one time i.e. perform multiplexing. These targets can be proteins or nucleic acid targets from different cellular species, such as for infectious disease diagnosis, or from the same species i.e. along the same genome, such as single-nucleotide polymorphisms (SNPs) genotyping for pharmacogenomics. It can also be for identifying aberrant biomolecules from normal ones, such as mutation detection in cancer diagnostics and prognostics. Therefore having a platform capable of performing multiplexed biological detection is an indispensable tool for accurate clinical diagnostics.

Through advancement in molecular biology as well as in areas such as microelectronics, microfabrication, material science, and optics, there have been a proliferation of miniaturized platforms, or biosensors, for performing biological analysis based on a variety of multiplexing technologies. These ranged from those capable of detecting a few targets to those capable of interrogating hundreds or even thousands of targets. Here we attempt to provide a concise overview of such technologies, as well as provide some insight into a simple technology that we developed in-house. Due to the enormous amount of progress in this area, this is by no means a comprehensive overview.

\section{Review of current technologies}

\subsection{Solution-based}

One of the most widely used technologies for multiplexed detection involves performing the detection within a single homogeneous solution. The best example of this is the multiplexed polymerase chain reaction (PCR). PCR, which is one of the most common techniques used in 
molecular biology, involves using a pair of primers to amplify a certain fragment of a target DNA or RNA manifold, until there is sufficient amount for detection or further downstream analysis. In multiplex PCR, several pairs of primers are used to simultaneously amplify different fragments. It is relatively easy to perform multiplexing in PCR, because the primers can first be designed to amplify fragments of different sizes, and these fragments can then be detected based on their size differences, either using gel electrophoresis or highresolution melting on real-time PCR systems. Alternatively, the different fragments can also be targeted by different probes conjugated to fluorescent dyes of a specific color. Upon hybridizing to the targets, the probes emit an optical signal corresponding to their dye, which is detected in a real-time PCR system.

Multiplex PCR is one of the most common techniques used in clinical diagnostics because the technology has matured significantly since its invention almost three decades ago. This is also rather easy to implement on biosensors, as the process can be carried out in microchambers (Merritt , 2010), or coupled to a capillary electrophoretic module (Thaitrong, 2009). The ability to perform multiplexed detection in PCR results from (a) the unique feature in PCR that allows primers to be designed to amplify fragments of different sizes, (b) the ability of the gel electrophoresis or real-time PCR system to differentiate the fragments by size as a result of their difference in electrophoretic mobility or melting temperature, and (3) the ability to differentiate the probes through color-emitting dyes. Probes used in multiplex PCR are conjugated with fluorescent dyes that emit different wavelengths of light, allowing them to be differentially detected. As a result, there is always a need for powerful optical detection, being capable of exciting and detecting one or multiple wavelengths of light. Due to limitations in the number of different wavelengths of light that can be excited and detected, the number of different multiplexed targets that can be detected in a single reaction is generally not high. One way to overcome this limitation is to combine multiplex PCR with other technologies, such as microarrays.

\subsection{2-D microarray}

The development of microarrays is driven by the demand for high throughput multiplexed analysis, such as the mapping of the human genome. This platform enables hundreds of thousands of proteins or DNA probes to be precisely immobilized onto designated locations within a microscopic area of a silicon or glass substrate (Ramsay, 1998; Schena et al, 1995), with the different probes identified through their unique locations. The proteins or oligonuleotides can be immobilized onto the surface using a high precision robotic arrayer or synthesized in-situ using light-directed chemistry. With such high density chips, it becomes possible to perform massively parallel interrogation of a large number of targets, making microarrays a platform of choice for applications such as gene expression analysis (Rahmatpanah, 2009), SNP genotyping (Wang et al, 1998; Lindroos et al, 2001) and transciptome analysis (Li et al, 2006).

Since the inception of the microarrays about two decades ago, there has been a host of companies offering the technology commercially. United States-based Affymetrix is one of the first companies to offer commercial oligonucleotide microarrays, with its GeneChip one of the most widely-used microarrays in a variety of applications, such as in prediction of tumour relapse in hepatocecullar carcinoma patients (Roessler, 2010). Other companies include Agilent, which uses inkjet printing for oligo synthesis on its 2D microarrays (Fig. 1), Applied Microarrays and Roche NimbleGen. CombiMatrix's CMOS arrays have addressable 
electrodes that have been developed for both DNA detection and immunoassays (Gunn, 2010; Cooper, 2010). With the advent of microfabrication technology and with increased competition, the prices of these microarrays have come down significantly over the years, making the technology more accessible to the research and clinical diagnostics community.
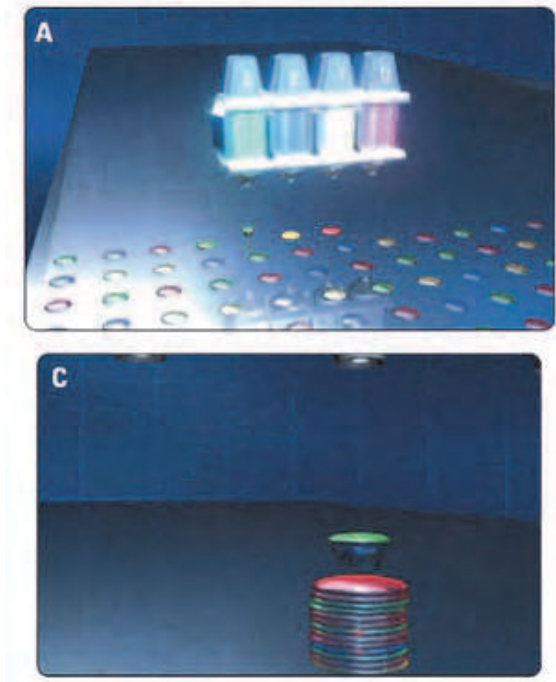
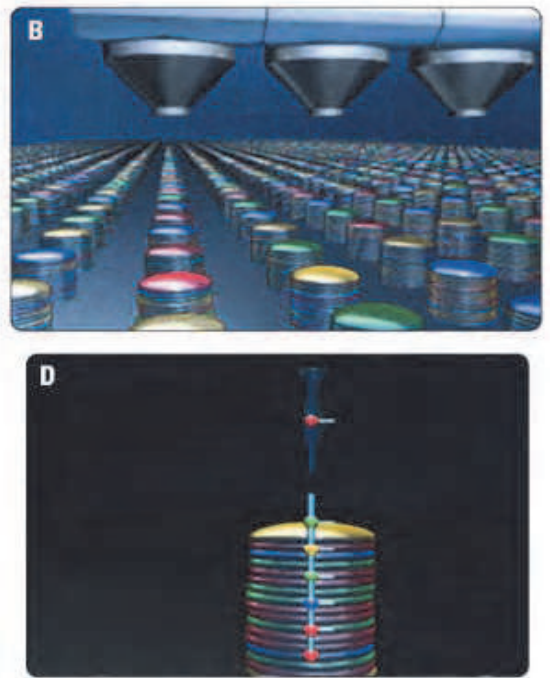

Fig. 1. Agilent's inkjet printing technology for oligonucleotide synthesis on 2D microarrrays A: the first layer of nucleotides is deposited on the activated microarray surface. B: growth of the oligos is shown after multiple layers of nucleotides have been precisely printed. C: close-up of one oligo as a new base is being added to the chain, which is shown in figure D. (Courtesy of Agilent Technologies. All rights reserved).

\subsection{3-D microarray}

Despite its high-throughput potential, the 2-D microarray format is restricted by the diffusion-limited kinetics, and electrostatic repulsion between the solution-phase targets and the densely localized solid-phase probes. Furthermore, the amount of probes that can be immobilized on the planar substrate, and hence the sensitivity and signal-to-noise ratio (SNR), is also somewhat limited. The introduction of 3-D microarrays go some way toward overcoming these limitations. These 3-D microarrays comprised of additional microstructures that are fabricated onto planar substrates to provide a high surface-density platform that increases the immobilization capacity of capture probes, enhances target accessibility and reduces background noise interference in DNA microarrays, leading to improved signal-to-noise ratios, sensitivity and specificity.

An example of an early 3-D microarray is the gel-based chip (Kolchinsky \& Mirzabekov, 2002). The use of an array of nanoliter-sized polyacrylamide gel pads on a glass slide provides distinct 3D microenvironments for the immobilization of oligonucleotides. Compared to planar glass substrates, the gel-based format can be applied with a higher probe concentration of up to 100 fold, thereby increasing the SNR. The near solution-phase interaction between targets and probes within individual gel pads can also potentially 
alleviate the problems associated with diffusion-limited kinetics. These gel-based microarrays have been successfully demonstrated for the detection of SNPs associated with $\beta$-thalassemia mutations (Drobyshev et al, 1997), and for the identification of polymorphisms in the human mu-opioid receptor gene (LaForge et al, 2000).

Other 3-D structures fabricated onto planar surfaces include conical dendrons as well as micropillars (Hong et al, 2005). By fabricating conical dendrons, nano-controlled spacings can be created to provide enough room for the target strand to access each probe, thereby creating a reaction format resembling that in a solution (Fig. 2). As a result, the hybridization time can be reduced to significantly to allow effective discrimination of singlenucleotide mismatches (Hong et al, 2005).

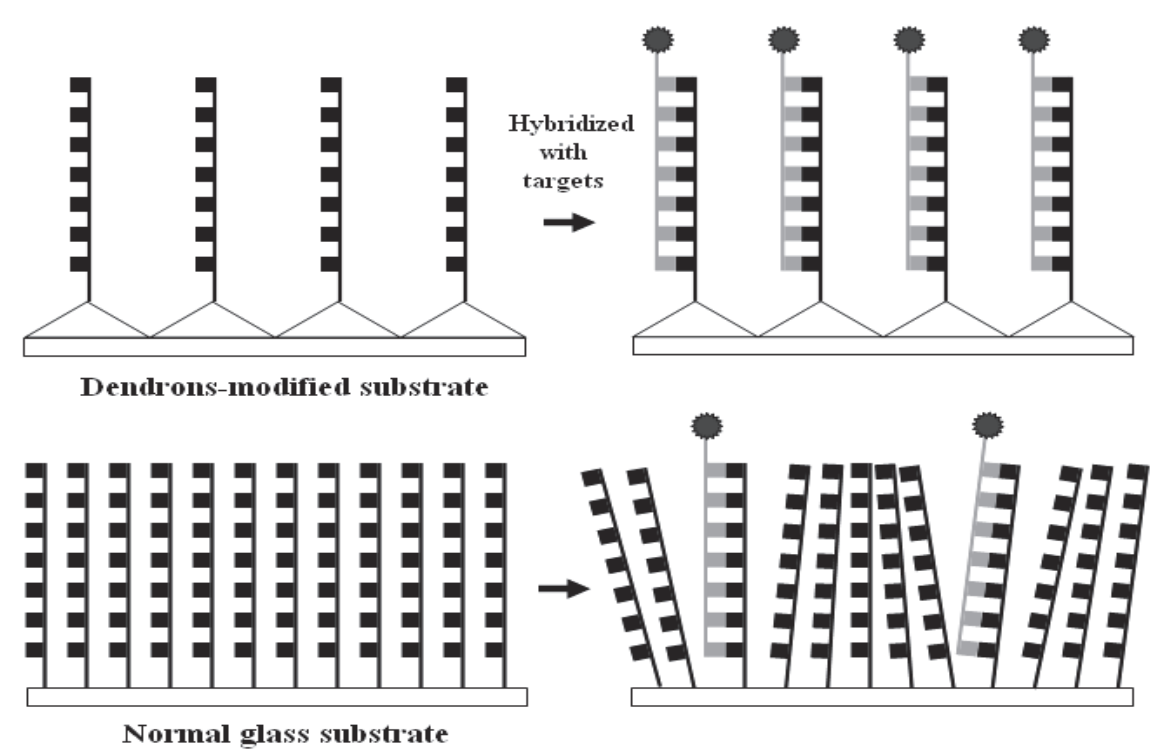

Fig. 2. Schematic diagram showing improved DNA hybridization onto a dendron-modified substrate as compared to that of a normal substrate.

Ramanamurthy et al (2008) reported the fabrication of ordered, high-aspect ratio nanopillar arrays on the surface of silicon-based chips to enhance signal intensity in DNA microarrays (Fig. 3). These 150-nm diameter nanopillars were found to enhance the hybridization signals by up to 7 times as compared to flat silicon dioxide substrates. In addition, hybridization of synthetic targets to capture probes that contained a single-base variation showed that the perfect matched duplex signals on dual-substrate nanopillars can be up to 23 times higher than the mismatched duplex signals. The Z-Slides microarray from United States-based company Life Bioscience comprises micropillars and nanowells to enhance spot morphology and eliminate cross-talk between probe sites. By detecting only the pillar surfaces which are several hundred microns from the base, background noise is removed from the microarray scan.

A 3-D microarray which is markedly different from the above-mentioned approaches involves immobilizing oligonucleotide probes onto a single thread instead of a planar 
substrate (Stimpson et al, 2004). The thread is subsequently wound around a core to form a compact, high-density SNP detection platform. Hybridization can be carried out by immersing the thread-and-core structure into a target solution, and completed within approximately $30 \mathrm{~min}$. This platform has been demonstrated for the analysis of SNPs in CYP2C19, an important cytochrome P450 gene (Tojo et al, 2005).

(a)

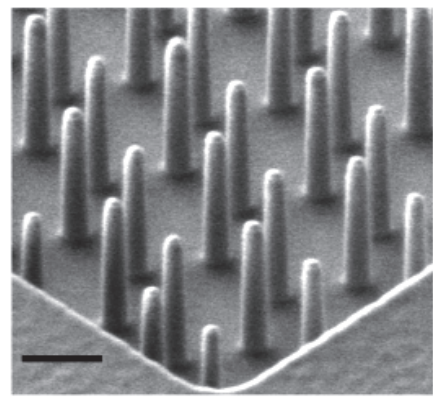

(c)

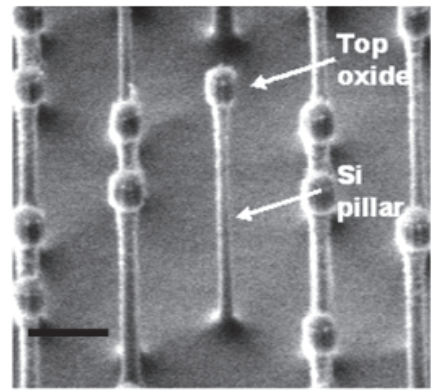

(b)

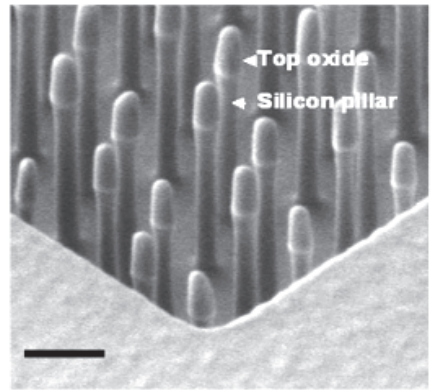

(d)

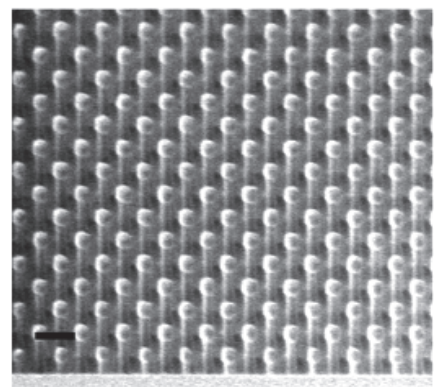

Fig. 3. SEM images of the nanopillars fabricated on silicon-based biosensors. (a) Singlesubstrate nanopillars consisting $\mathrm{SiO}_{2}$. (b) Dual-substrate nanopillars consisting $\mathrm{SiO}_{2}$ layer atop the Si pillar. (c) Very high-aspect ratio dual-substrate nanopillars. (d) Dense array of ordered dual-substrate nanopillars. Scale bars are all $500 \mathrm{~nm}$.

\subsection{Bead microarray}

One of the best examples of 3-D microarrays, and perhaps also one of the most successful commercially available platforms, is the bead microarray. Unlike 2-D microarrays, the high surface-to-volume ratio of beads allows a larger amount of probes to be immobilized to improve the detection signals and signal-to-noise ratios. The small size of beads can further reduce the reaction volume, and the use of microfluidics in bead arrays can shorten the hybridization time to $<10 \mathrm{~min}$, a 50 to 70 -fold reduction as compared to conventional microarrays (Ali et al, 2003). Unlike 2-D or the 3-D microarrays discussed, probes are usually conjugated onto the beads prior to them being immobilized onto the microarrays. The major challenge, therefore, in developing bead arrays is to identify the identities or their corresponding immobilized probes of those randomly assembled beads in multiplexed analyses.

The most common strategy is to encode beads with colorimetric signatures using semiconductor nanocrystals, visible dyes or fluorophores, and subsequently decode them 
through visual or fluorescence detection (Mulvaney et al, 2004). Color-encoded beads are produced by embedding them with semiconductor nanocrystals, visible dyes, or fluorophores and subsequently decoded through visual or fluorescence detection. For example, Li et al (2001) mixed blue, green and orange fluorophores to yield 39 different codes for encoding $3.2 \mu \mathrm{m}$-diameter polystyrene beads assembled onto a wafer. Alternatively, two fluorophores can be mixed in different proportions to yield 100 distinguishable bead types that are subsequently decoded using two laser beams, as in the Luminex xMAP technology (Dunbar, 2006) (Fig. 4). The emission characteristics of organic fluorescent dyes are affected by changes in temperature, which may result in some bias when used in temperature-dependent studies (Liu et al, 2005). The fluorescent dyes also suffer from photobleaching and this can significantly affect the discriminability between color codes, particularly if they are distinguished by the difference in their intensities.

Quantum dots, which are photostable, have size-tunable emission wavelengths, and can be excited by a single wavelength to emit different colors at one time, are widely used to distinguish beads. Han et al. (2001) incorporated quantum dots at different intensities and colors to yield spectrally distinguishable polymeric beads of up to 10 distinct types (Fig. 4). Using 5-6 colors, each at 6 intensity levels, it is possible to achieve up to 40000 codes using this approach, although this has yet to be demonstrated. These techniques for color encoding beads are straightforward in that the color-emitting agents are directly impregnated into the beads. However, this also means that the encoder signals cannot be removed, resulting in possible interference between the encoder and reporter signals. To avoid this, the number of reporter dyes available for use would inadvertently be reduced. Also, encoding the beads into unique color codes is challenging as the color-emitting agents must be mixed in precise proportions. The difficulty in distinguishing a large number of color codes further means that only up to 100 color codes have been demonstrated so far, limiting them to low or medium throughout applications (Xu et al, 2003; Li et al, 2004).
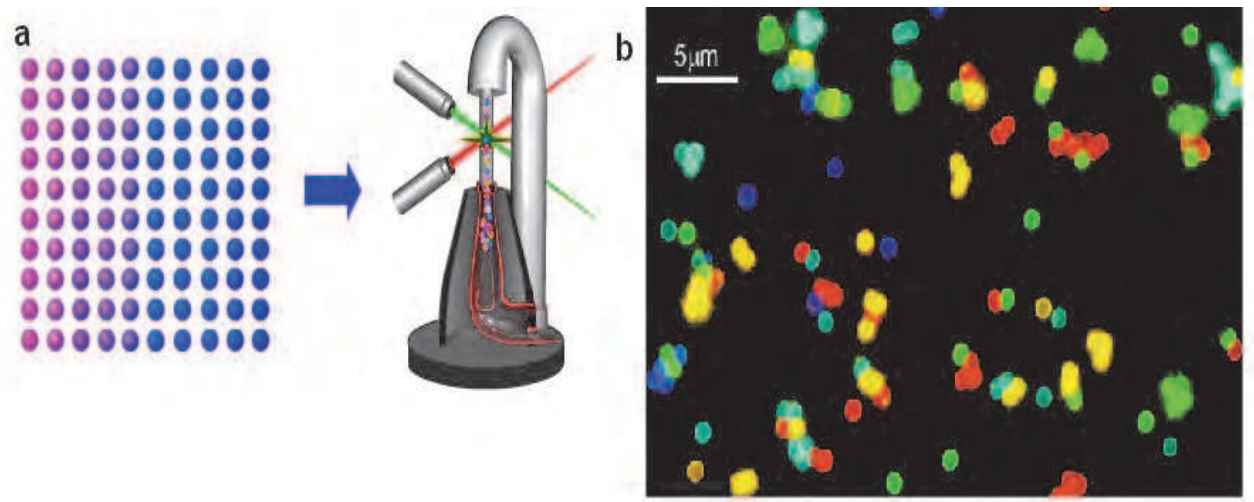

Fig. 4. (a) A set of 100 distinguishable bead types can be created by mixing precise proportions of two fluorescent dyes, and subsequently detected using a flow cytometer with two laser beams. (Courtesy of Luminex Corporation. All rights reserved). (b) Quantum dot nanocrystals of 10 different emission colors incorporated into the beads to create spectrally distinguishable types. (Adapted by permission from Macmillan Publishers Ltd: Nature Biotechnology, copyright 2001). 
Beads within an array can also be individually addressed using barcodes. A graphical barcode can also be written inside fluorescently dyed beads through a technique termed "spatial selective photobleaching of the fluorescence" (Braeckmans, 2001). Using a specially adapted laser scanning confocal microscope, any sort of pattern can be photobleached at any depth inside the fluorescently dyed bead. This technique was used to photobleach a barcode of different band widths onto $45 \mu \mathrm{m}$-diameter fluorescent beads. The advantages of this technique are that only a single fluorescent dye is needed in the encoding scheme, and the number of codes achievable is virtually unlimited. However, there is still the problem of interference between the encoder and reporter fluorescence signals, while the effects of photobleaching during the decoding stage might alter or degrade the barcode.

A widely used bead microarray platform for biological detection and clinical diagnostics is the commercial BeadArray from Illumina, a market leader in high-throughput bead microarrays. It assembles 3-micron silica beads onto a fiber optic of planar silica slides, for a range of DNA and RNA analyses. There is also the Veracode technology, which uses digital holographic barcode to identify the beads (Lin et al, 2009) (Fig. 5). When excited by a laser, each microbead, which has a pillar-like rather than spherical shape, emits an image resembling a barcode. Using this method, it becomes possible to have virtually unlimited number of different bead types. The platform can be applied to both protein-based or DNAbased assays.

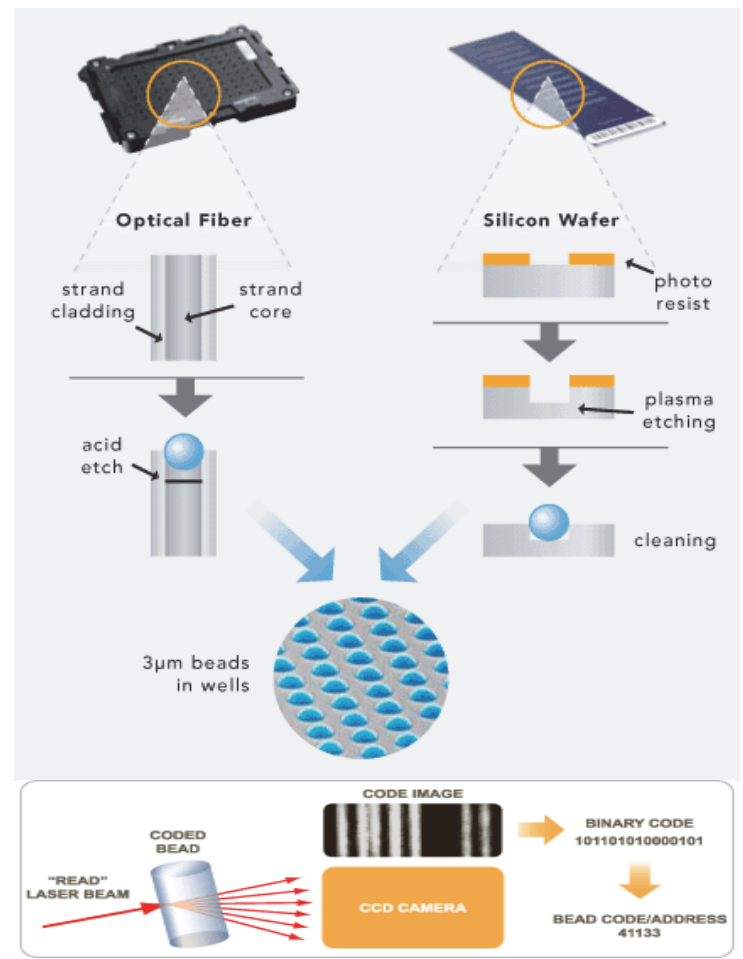

Fig. 5. Illumina's BeadArray (top panel) and Veracode technology (bottom panel). (Courtesy of Illumina. All rights reserved) 


\section{A simple spatially addressable bead-based biosensor}

We describe here some of our own work in developing a biosensor that allow different bead types to be incorporated and addressed with minimal efforts for encoding and decoding, simplifying the development and usage of such devices ( $\mathrm{Ng}$ et al, 2010). To achieve that, different bead types are incorporated and identified based on their spatial addresses (akin to microarrays) without the need for color-coding (Fig. 6). Beads of a certain type are spotted onto a polymeric micro-matrix (or gel pad) fabricated on the surface of the biosensor. The natural immobilization of the beads by the gel pad allows each bead to be anchored within the gel pad on a unique location, acquiring spatial addresses that can be easily recorded via an acquired image. Beads of a second type spotted over the same gel pad take up spatial addresses distinct from those of the first bead type, allowing the two bead types to be easily distinguished. This is repeated for immobilizing and distinguishing further bead types on the gel pad, obviating the need for prior encoding and tedious decoding of beads. The throughput can be increased by further spotting many different bead types onto the hundreds of gel pads on each biosensor. We demonstrate the use of this bead-based biosensor for detection of six common South-east Asian beta-globin gene mutations within $30 \mathrm{~min}$, demonstrating its potential as a simple tool for rapid beta-thalassemia carrier screening.

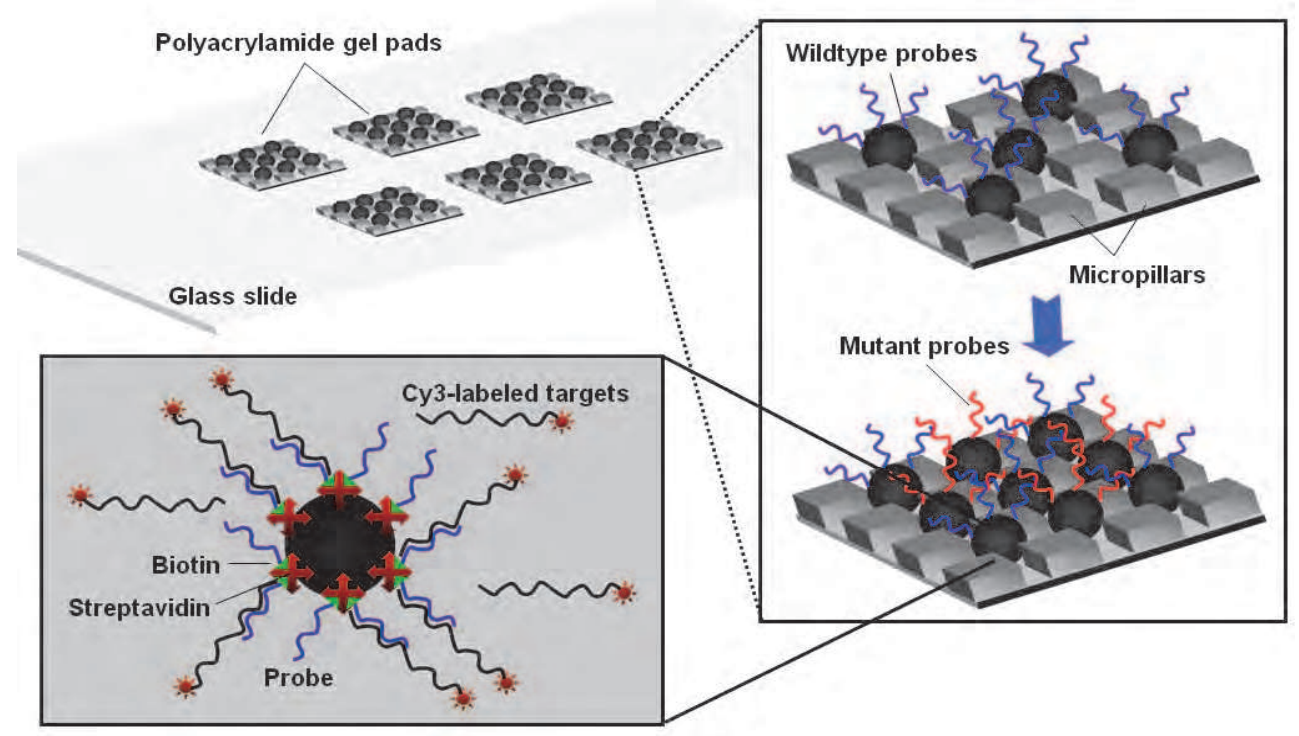

Fig. 6. Schematic representation of the spatially addressable bead-based biosensor (Adapted from $\mathrm{Ng}$ et al, 2010, copyright Elsevier Inc).

\subsection{Biosensor fabrication}

The biosensor consisted an array of $19 \times 24$ polyacrylamide gel pads fabricated on a glass slide (Corning, Corning, NY) pre-treated with Bind Silane (GE Healthcare, Piscataway, NJ). 
The gel pads had horizontal and vertical pitch of $300 \mu \mathrm{m}$, and each gel pad further comprised a $10 \times 10$ array of micropillars $(10 \times 10 \times 10 \mu \mathrm{m})$ with horizontal and vertical pitch of $10 \mu \mathrm{m}$ (Fig. 7). A photopolymerization process described previously was used to create the array of gel pads (Proudnikov et al., 1998), after which the glass slide was treated in 0.1M $\mathrm{NaBH}_{4}$ for 30 min to reduce gel pads auto-fluorescence.

The Biochip Arrayer (PerkinElmer, Boston, MA) was used to spot beads onto singular gel pads on the device. Each gel pad was spotted with about $5 \mathrm{~nL}$ of a particular bead solution ( $\sim 9000$ beads $/ \mu \mathrm{L}$ ), and then left to dry at room temperature for 2-3 min to allow beads immobilization to the gel. Beads can also be spotted manually using a pipette, although this required a larger amount of bead solution $(0.25 \mu \mathrm{L})$ per spot and the beads usually covered 2-4 gel pads simultaneously. Positions of each spotted bead type were then recorded via autofluorescence images for determining their spatial addresses. This was repeated until all bead types for detecting a particular target were immobilized on the same gel pad. The device can then be capped with a microfluidic module for sample flow-through, or the buffer can also be applied over the spotted beads without the module. The polydimethylsiloxane (PDMS) module was fabricated using common soft lithographic techniques (Duffy et al., 1998).

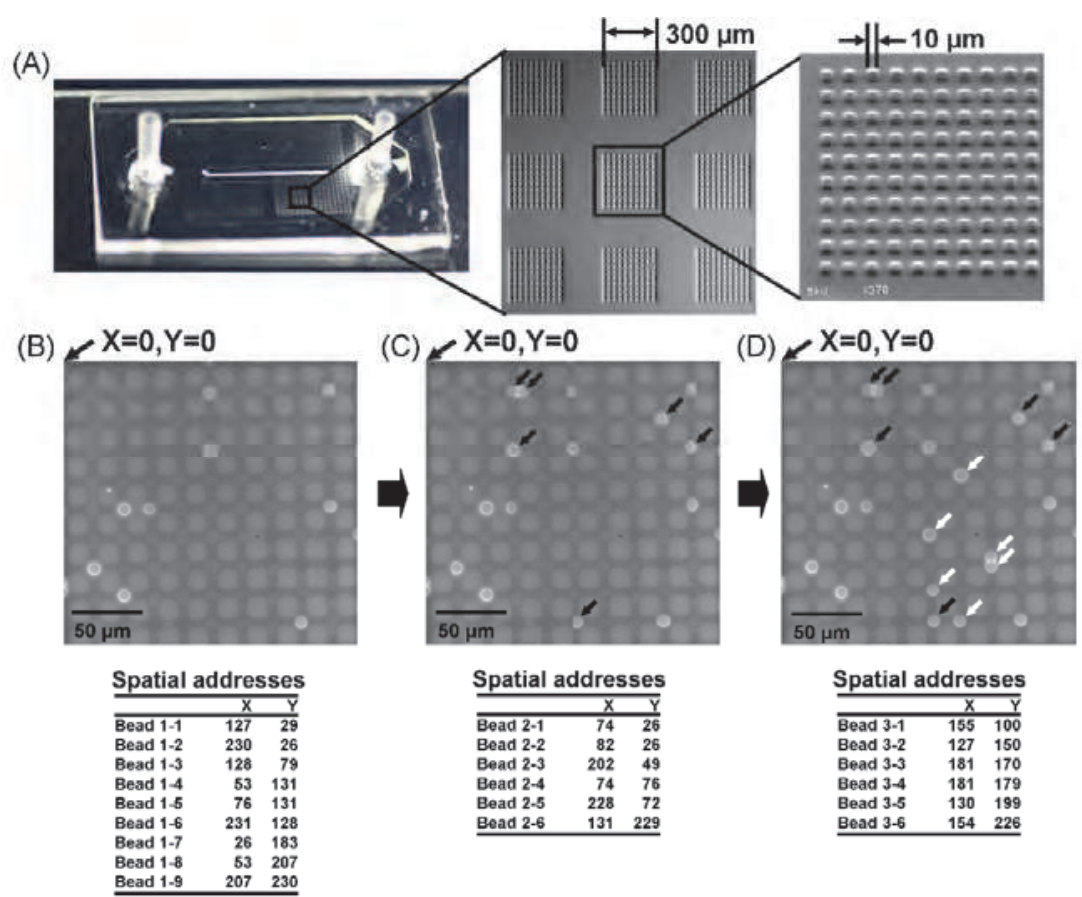

Fig. 7. The bead-based biosensor. (A) The device comprised an array of polyacrylamide gel pads on a glass slide. Each gel pad further comprised an array of micropillars. (B) Image after spotting the first bead type onto the gel pad. The spatial address for each bead is recorded in terms of their $x, y$ coordinates. (C) Image after spotting a second bead type (black arrows) and finally (D) a third bead type (white arrows). (Adapted from $\mathrm{Ng}$ et al, 2008, copyright Elsevier Inc). 


\subsection{Oligonucleotide probes and targets}

The six common South-east Asian beta-globin gene mutations selected for this study were $28 \mathrm{~A} \rightarrow \mathrm{G},-29 \mathrm{~A} \rightarrow \mathrm{G}$, IVSI5 $\mathrm{G} \rightarrow \mathrm{C}$, IVSI1 G $\rightarrow$ T, Cd26 GAG $\rightarrow$ AAG, and IVSII654 $\mathrm{C} \rightarrow \mathrm{T}$. For each mutation, allele-specific probes were designed to hybridize with perfect complementary to either the wildtype or mutant variant (Table 1). A biotin moiety was added to the $5^{\prime}$ end of each probe, and conjugation of probes to $9.95 \mu \mathrm{m}$ streptavidinmodified polystyrene beads was carried out according to previously described protocol $(\mathrm{Ng}$ et al., 2008).

PCR was carried out to amplify two fragments of the beta-globin gene, with the first fragment (319 bp) encompassing the Exon 1 which incudes all the targeted mutations other than IVSII654 C $\rightarrow \mathrm{T}$, which was contained in the second fragment (128 bp). Primer sequences were: Frag1-F: 5'-Cy3-ACggCTgTCATCACTTAgAC-3' (Genbank HUMHBB sequence 62010-62029); Frag1-R: 5'-CCCAgTTTCTATTggTCTCC-3' (HUMHBB sequence 6232862309); Frag2-F: 5'- Cy3-TgTATCATgCCTCTTTgCACC-3' (HUMHBB sequence 6322763247); and Frag2-R: 5'-CAATATgAAACCTCTTACATCAg-3' (HUMHBB: 63354-63332).

Genomic DNA (100 ng) was amplified in a total volume of $50 \mu \mathrm{L}$ containing $0.5 \mu \mathrm{M}$ each of the two sets of primers, $200 \mu \mathrm{M}$ of each deoxynucleotide triphosphate, and $1 \mathrm{U}$ of HotStarTaq DNA polymerase in $1 \times$ supplied PCR buffer (Qiagen). Amplification was carried out in an iCycler thermal cycler (BioRad) with an initial denaturation at $95{ }^{\circ} \mathrm{C}$ for 15 min, followed by 35 cycles at $98{ }^{\circ} \mathrm{C}$ for $30 \mathrm{~s}, 55^{\circ} \mathrm{C}$ for $30 \mathrm{~s}$, and $72{ }^{\circ} \mathrm{C}$ for $30 \mathrm{~s}$, and a final extension at $72{ }^{\circ} \mathrm{C}$ for $5 \mathrm{~min}$. Products were then re-amplified with only the forward primers to generate ssDNA for allele-specific hybridization.

\begin{tabular}{|c|c|c|}
\hline Probe name & Mutation targeted & Sequence $\left(5^{\prime}-3^{\prime}\right)$ \\
\hline$-28,-29 \_W T$ & $-28 /-29 W T$ & CCTgACTTTTATgCCCAg \\
\hline -28_MT & $-28 \mathrm{MT}$ & СCTgACTTCETATgCCCAg \\
\hline -29_MT & $-29 \mathrm{MT}$ & CCTgACTTTCEATgCCCAg \\
\hline IVSI5,1_WT & IVSI5/1 WT & CTTgATACCAACCTgCCC \\
\hline IVSI5_MT & IVSI5 MT & CTTgATAgCAACCTgCCC \\
\hline IVSI5_WT & IVSI1 MT & CTTgATACCAA믁ㄷСC \\
\hline Cd26_WT & Cd26 WT & gggCСТCACCACCAAC \\
\hline Cd26_MT & $\mathrm{Cd} 26 \mathrm{MT}$ & gggCCTIACCACCAAC \\
\hline IVSII654_WT & IVSII654 WT & TTgCТАTТgССТТААССС \\
\hline IVSII654_MT & IVSII654 MT & TTgCТАТТAСССТТАACСС \\
\hline
\end{tabular}

WT: wild-type, MT: mutant

Table 1. Probe sequences for targeting each of the beta-globin gene mutations selected for this study. (Adapted from Ng et al, 2010, copyright Elsevier Inc).

\subsection{Hybridization and signal detection}

Re-amplified PCR products were purified using the Microcon YM-30 filter device (Millipore) before being diluted to a $10 \mu \mathrm{L}$ hybridization solution containing $500 \mathrm{mM} \mathrm{NaCl}$ and $30 \%$ formamide. Hybidization was carried out by pipetting the solution over the spotted beads. After 30 min incubation, the device was rinsed briefly with a solution 
containing only $500 \mathrm{mM} \mathrm{NaCl}$ and $30 \%$ formamide, and signal capture was carried out by fluorescence imaging. The imaging system comprised an epifluorescence microscope (BX51, Olympus), $100 \mathrm{~W}$ mercury lamp and fluorescence filter set 41007 (Chroma Technology). MetaMorph 5.0 (Molecular Devices) was used to control acquisition of 12-bit monochrome bead images at $2 \mathrm{~s}$ exposure from a SPOT-RT Slider cooled-CCD camera (Diagnostic Instruments), and bead signals were quantitated using the modified version of a software developed in-house previously (Ng and Liu, 2005).

\subsection{Results and discussion}

To demonstrate detection of the six beta-globin gene mutations, six human samples heterozygous for $-28 \mathrm{~A} \rightarrow \mathrm{G},-29 \mathrm{~A} \rightarrow \mathrm{G}$, IVSI5 $\mathrm{G} \rightarrow \mathrm{C}$, IVSI1 $\mathrm{G} \rightarrow \mathrm{T}, \mathrm{Cd} 26 \mathrm{GAG} \rightarrow \mathrm{AAG}$, and IVSII654 $\mathrm{C} \rightarrow \mathrm{T}$, and one homozygous for IVSII654 $\mathrm{C} \rightarrow$ T were analyzed using the bead-based biosensor. All samples were genotyped previously by direct sequencing or multiplexed minisequencing (Wang et al., 2003). Wildtype and mutant probes targeting each mutation were conjugated to distinct bead sets, spotted onto a particular gel pad on the device, and distinguished based on their spatial addresses (Fig. 8A). Probes were designed with the targeted mutation as near as possible to its centre region, in order to increase the discrimination between matched and mismatched duplexes. Due to the proximity between the -28 and -29 mutations, as well as between the IVSI1 and IVSI5 mutations, each pair of mutations must be detected simultaneously on a single gel pad by four sets of probes to cover all possible genotypes. However, due to the lack of samples compound heterozygous for $-28 /-29$ and IVSI1/IVSI5, only three sets of probes were required in this study for each pair of mutations.

Fig. 8B shows the signal intensity from the wildtype and mutant probes used to target each mutation. All seven different samples were correctly genotyped using the device. For heterozygous mutations, signal intensities from the wildtype probes did not differ significantly from that of the mutant probes, attaining student t-test $p$-values $>0.05$ for all except IVSII654 which had a slightly lower p-value of about 0.01 . In the absence of a mutation, the wildtype probe intensities were significantly higher than that of the mutant probes, with p-values far lower than 0.001. For the homozygous IVSII654 mutation, the mutant probe intensity was significantly higher than the wildtype probe, attaining a p-value $<0.0001$. This similarity or significant difference between wildtype and mutant probe intensities allowed correct identification of the heterozygous mutant and homozygous wildtype (or mutant) samples respectively.

The spatially addressable bead-based biosensor offers an alternative tool for simple yet efficient and rapid detection of beta-thalassemia mutations. The device is comprised simply of a glass slide fabricated with a thin polyacrylamide matrix on its surface using a photopolymerization process that is faster $(\sim 45 \mathrm{~min})$ and far less complicated than conventional photolithographic techniques for making silicon chips. The main advantage of the device is its ability to distinguish different bead types without the need for prior timeconsuming and laborious techniques such as color-encoding (Braeckmans et al., 2002). This is due to the natural immobilization of the beads to the polyacrylamide gel pads, thus allowing the beads to acquire unique spatial addresses. Detection is achieved by applying the solution of PCR-amplified targets over the region of the spotted beads for passive hybridization to occur, which obviates the need for microfluidic mixing and thus microchannels. This further simplifies the fabrication process, lowers the cost of the device, 
(A)

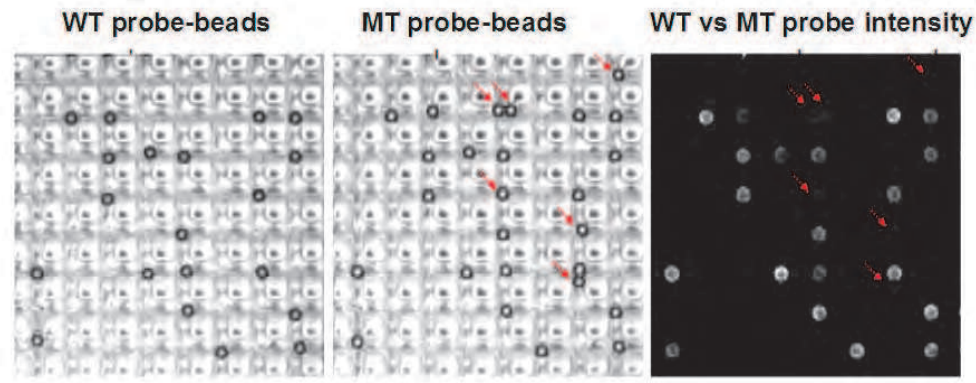

(B)
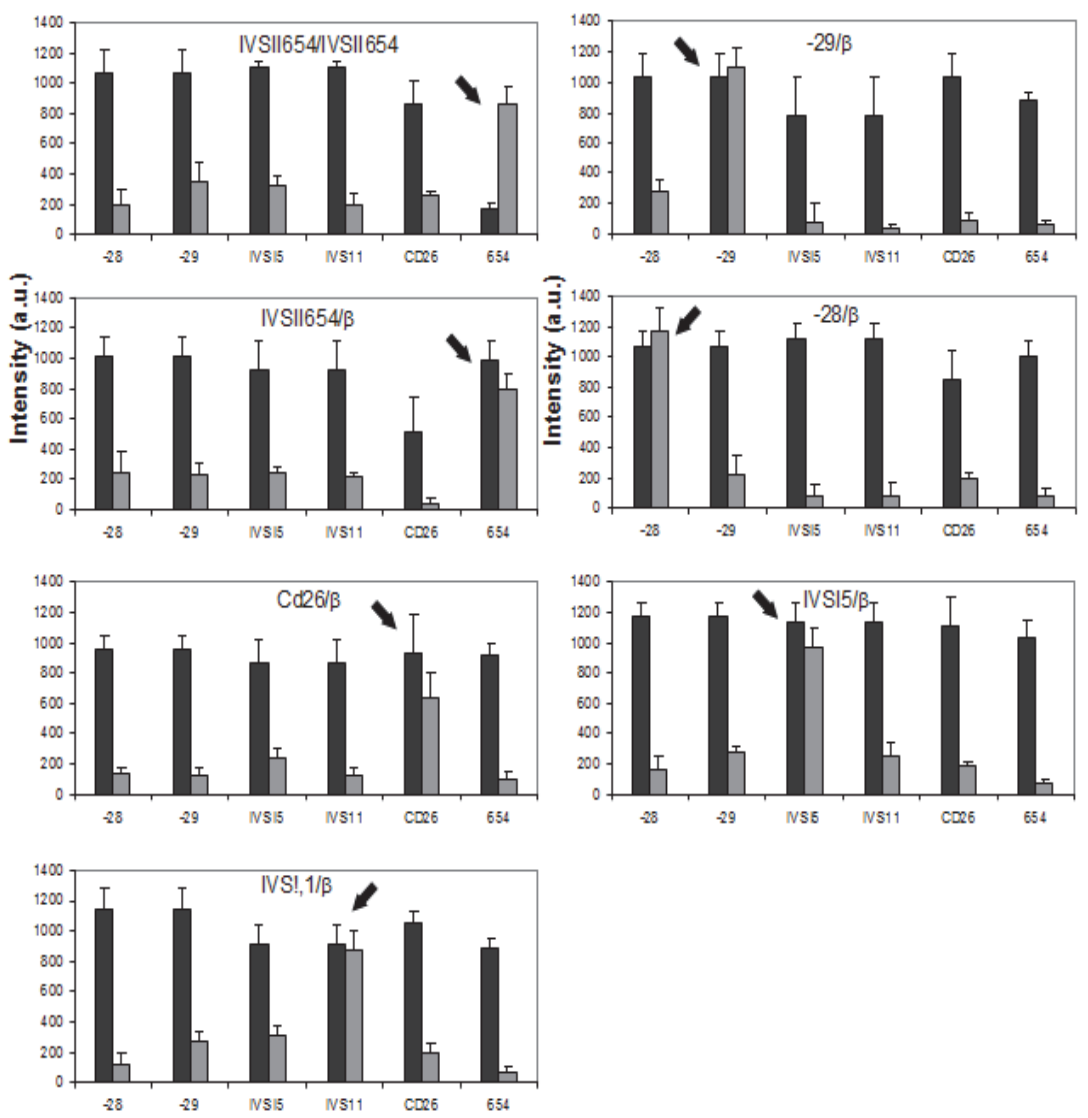

Fig. 8. Allele-specific hybridization on the device. (A) Typical example of the beads spotted onto a gel pad. Probe-beads targeting Cd26 wildtype variant were spotted onto a gel pad, followed by those targeting the mutant variant (red arrows). Difference in probe intensities showed sample to be of homozygous Cd26 normal genotype. (B) Signal intensity from the wide-type ( $\mathbf{})$ and mutant ( $\mathbf{})$ probe-bead targeting each of the six mutations selected for this study. (Adapted from Ng et al, 2010, copyright Elsevier Inc). 
and reduces the sample volume required $(<10 \mu \mathrm{L})$. Despite the lack of microfluidic mixing, detection is achieved in $30 \mathrm{~min}$, although this might possibly be even faster, given that we have achieved hybridization on this device within $10 \mathrm{~min}$, albeit with synthetic targets $(\mathrm{Ng}$ et al., 2008).

\section{Conclusion}

The advent of biosensors has allowed biomedical research and clinical diagnostics to leverage upon the advantages of miniaturization, such as reduced sample volumes, faster reaction times, and the possibility of multiplexed detection. The last point is of particular importance, since the simultaneous detection of multiple targets at once has resulted in significant time savings, particularly for applications requiring high-throughput. Often, multiple targets must be detected in order to draw any meaningful conclusion in clinical diagnosis. So much progress has been made in this field such that it is now possible to utilize high throughput platforms such as microarrays to interrogate thousands of targets at once. The crucial role played by these technologies, such as multiplex PCR and the various forms of 2D, 3D and bead-based microarrays, in the past decades is indisputable, and will continue to be so. However several challenges exist.

First, it is important to reduce the cost of some of these technologies so as to make it more affordable, particularly for clinical diagnostics. For example, systems for real-time PCR can be quite costly, due in part to the high precision optical detection modules found within. With advances in optics, both light sources (e.g. LEDS) and detectors (e.g. digital cameras) are getting more affordable, which would help to bring down the costs of such systems. Also, part of the costs are attributable to the licensing issues. Manufacturers of real-time PCR systems and reagents have to pay a license fee including royalties to the original patent owners. With time, some of the patent protections will expire soon, so prices should also come down, as in the case of the patent expiry of the Taq polymerase in 2006. The manufacturing costs for microarrays and its bead-based counterpart are also high. Hopefully with advances in manufacturing technologies, the cost can eventually be reduced. Second, it is important for these technologies to be of sufficient sensitivity and specificity in order to meet the standards required in clinical diagnostics. Real-time PCR has no problems with that, since it is not uncommon for it to achieve a sensitivity and specificity close to $100 \%$. 2-D microarrays, on the other hand, might face more of a challenge. The diffusionlimited kinetics, steric hindrances and high noise contributed by the planar surface might somewhat affect sensitivity and specificity. It is important to ascertain that the microarrays can reproducibly meet the required levels of sensitivity and specificity before its application to clinical diagnostics.

Third, the reaction times for some applications can still be rather high, particularly for the microarrays. It is desirable to reduce these times further since clinical diagnostics often require a fast turn around time to minimize patient anxiety and to aid decision making in disease management.

Finally, with the advent of modern technologies, some of the multiplexing technologies discussed here might find themselves being slowly displaced. Sequencing is a method used to decipher the order of bases along a DNA. Traditionally slow, it is now possible to perform massively parallel sequencing on high-throughput platforms to speed up its rate. Known as next generation sequencing, thousands of sequences can now be generated at once, using commercial sequencers from companies such as Illumina (Solexa), Roche (454) 
and Applied Biosystems. Some of these platforms, like the SOLiD system from Applied Biosystems, can generate up to 60 gigabases of DNA sequence per run. With these advances in next generation sequencing comes the race for rapid and low cost full genome sequencing. The Archon X Prize for Genomics was established in October 2006 to award US $\$ 10$ million to "the first Team that can build a device and use it to sequence 100 human genomes within 10 days or less, with an accuracy of no more than one error in every 100,000 bases sequenced, with sequences accurately covering at least $98 \%$ of the genome, and at a recurring cost of no more than $\$ 10,000$ per genome". As of January 2011, the prize is yet unclaimed. However, the possibility of being able to sequence an entire human genome accurately, cheaply and rapidly in future might supplant some of today's multiplexing technologies like the DNA microarray.

In summary, multiplexing capabilities in biosensors have come a long way and will continue to advance rapidly in the next decade, with a large number of companies pouring in large sums of monies into research and development. The ideal platform will be one offering high-throughput, rapid and low cost diagnostics. Whether that can be realised in the near future remains to be seen.

\section{References}

Ramsay, G. (1998). DNA chips: State-of-the art. Nat Biotech 16, 40.

Ali, M.F., Kirby, R., Goodey, A.P., Rodriguez, M.D., Ellington, A.D., Neikirk, D.P. \& McDevitt, J.T. (2003). DNA hybridization and discrimination of single-nucleotide mismatches using chip-based microbead arrays. Anal Chem 75, 4732-4739.

Braeckmans, K. (2001). A new generation of encoded microcarriers. Drug Discovery Technology 12-17 Aug, Boston

Chen, J., Iannone, M.A., Li, M.-S., Taylor, J.D., Rivers, P., Nelsen, A.J., Slentz-Kesler, K.A., Roses, A. \& Weiner, M.P. (2000). A Microsphere-Based Assay for Multiplexed Single Nucleotide Polymorphism Analysis Using Single Base Chain Extension. Genome Res. 10, 549-557.

Cooper, J., Yazvenko, N., Peyvan, K., Maurer, K., Taitt, C.R., Lyon, W. \& Danley, D.L. (2010). Targeted deposition of antibodies on a multiplex CMOS microarray and optimization of a sensitive immunoassay using electrochemical detection. PLoS One. 19, e9781.

Daelemans C, Ritchie ME, Smits G, Abu-Amero S, Sudbery IM, Forrest MS, Campino S, Clark TG, Stanier P, Kwiatkowski D, Deloukas P, Dermitzakis ET, Tavaré S, Moore GE, Dunham I. (2010). High-throughput analysis of candidate imprinted genes and allele-specific gene expression in the human term placenta. BMC Genet. 19, 25

Drobyshev, A., Mologina, N., Shik, V., Pobedimskaya, D., Yershov, G. \& Mirzabekov, A. (1997). Sequence analysis by hybridization with oligonucleotide microchip: identification of beta-thalassemia mutations. Gene 188, 45-52.

Duffy, D.C., McDonald, J.C., Schueller, O.J.A. \& Whitesides, G.M. (1998). Rapid Prototyping of Microfluidic Systems in Poly(dimethylsiloxane). Anal. Chem. 70, 4974-4984.

Dunbar, S.A. (2006). Applications of Luminex(R) xMAP(TM) technology for rapid, highthroughput multiplexed nucleic acid detection. Clinica Chimica Acta 363, 71.

Gunn, S., Yeh, I.T., Lytvak, I., Tirtorahardjo, B., Dzidic, N., Zadeh, S., Kim, J., McCaskill, C., Lim, L., Gorre, M., Mohammed, M. (2010). Clinical array-based karyotyping of 
breast cancer with equivocal HER2 status resolves gene copy number and reveals chromosome 17 complexity. BMC Cancer. 28, 396

Han, M., Gao, X., Su, J.Z. \& Nie, S. (2001). Quantum-dot-tagged microbeads for multiplexed optical coding of biomolecules. Nat Biotechnol 19, 631-635.

Hong, B.J., Oh, S.J., Youn, T.O., Kwon, S.H. \& Park, J.W. (2005). Nanoscale-controlled spacing provides DNA microarrays with the SNP discrimination efficiency in solution phase. Langmuir 21, 4257-4261.

Hong, B.J., Sunkara, V. \& Park, J.W. (2005). DNA microarrays on nanoscale-controlled surface. Nucl. Acids Res. 33, e106.

Iannone, M.A., Taylor, J.D., Chen, J., Li, M.S., Rivers, P., Slentz-Kesler, K.A. \& Weiner, M.P. (2000). Multiplexed single nucleotide polymorphism genotyping by oligonucleotide ligation and flow cytometry. Cytometry 39, 131-140 .

Kolchinsky, A. \& Mirzabekov, A. (2002). Analysis of SNPs and other genomic variations using gel-based chips. Hum Mutat 19, 343-360 .

LaForge, K.S., Shick, V., Spangler, R., Proudnikov, D., Yuferov, V., Lysov, Y., Mirzabekov, A. \& Kreek, M.J. (2000). Detection of single nucleotide polymorphisms of the human mu opioid receptor gene by hybridization or single nucleotide extension on custom oligonucleotide gelpad microchips: potential in studies of addiction. Am J Med Genet 96, 604-615.

Li, A.X., Seul, M., Cicciarelli, J., Yang, J.C. \& Iwaki, Y. (2004). Multiplexed analysis of polymorphisms in the HLA gene complex using bead array chips. Tissue Antigens 63, 518-528.

Li, Y., Elashoff D., Oh, M., Sinha, U., St John, M.A., Zhou, X., Abemayor, E., \& Wong, D.T. (2006). Serum circulating human mRNA profiling and its utility for oral cancer detection. J Clin Oncol. 24, 1754.

Lin, C.H., Yeakley, J.M., McDaniel, T.K. \& Shen, R. (2009). Medium- to high-throughput SNP genotyping using VeraCode microbeads. Methods Mol Biol. 496, 129-42

Lindroos, K., Liljedahl, U., Raitio, M. \& Syvanen, A.C. Minisequencing on oligonucleotide microarrays: comparison of immobilisation chemistries. Nucleic Acids Res 29, E69-69 (2001).

Liu, W.-T., Wu, J.-H., Li, E.S.-Y. \& Selamat, E.S. (2005). Emission Characteristics of Fluorescent Labels with Respect to Temperature Changes and Subsequent Effects on DNA Microchip Studies. Appl. Environ. Microbiol. 71, 6453-6457.

Merritt, A.J., Keehner, T., O'Reilly, L.C., McInnes, R.L. \& Inglis, T.J. (2010). Multiplex amplified nominal tandem-repeat analysis (MANTRA), a rapid method for genotyping Mycobacterium tuberculosis by use of multiplex PCR and a microfluidic laboratory chip. J Clin Microbiol. 48, 3758-61

Mulvaney, S.P., Mattoussi, H.M. \& Whitman, L.J. (2004). Incorporating fluorescent dyes and quantum dots into magnetic microbeads for immunoassays. Biotechniques 36, 602606, 608-609.

Ng, J.K. \& Liu, W.T. (2005). LabArray: real-time imaging and analytical tool for microarrays . Bioinformatics. 21, 689-690.

Ng, J.K., Selamat, E.S. \& Liu, W.T. (2008). A Spatially Addressable Bead-based Biosensor for Simple and Rapid DNA Detection. Biosens Bioelectron. 23, 803-810. 
Ng, J.K., Wang, W., Liu, W.T., \& Chong, S.S. (2010). Spatially addressable bead-based biosensor for rapid detection of beta-thalassemia mutations. Anal Chim Acta. 658: 193-196.

Proudnikov, D., Timofeev, E. \& Mirzabekov, A., (1998). Immobilization of DNA in polyacrylamide gel for the manufacture of DNA and DNA-oligonucleotide microchips. Anal Biochem. 259, 34-41.

Rahmatpanah, F.B., Carstens, S., Hooshmand, S.I., et al. (2009). Large-scale analysis of DNA methylation in chronic lymphocytic leukemia. Epigenomics. 1, 39

Ramanamurthy, B., Ng, K.K.J., Shah, E.S., Balasubramaniam, N., and Liu, W.T. (2008). Silicon nanopillars substrate for enhancing signal intensity in DNA microarrays. Biosensors and Bioelectronics. 24, 723

Roessler, S., Jia, H.L., Budhu, A., Forgues, M., Ye, Q.H., Lee, J.S., Thorgeirsson, S.S., Sun, Z., Tang, Z.Y., Qin, L.X. \& Wang, X.W. (2010). A Unique Metastasis Gene Signature Enables Prediction of Tumor Relapse in Early-Stage Hepatocellular Carcinoma Patients Cancer Res 70, 10202-10212.

Schena, M., Shalon, D., Davis, R.W. \& Brown, P.O. (1995). Quantitative monitoring of gene expression patterns with a complementary DNA microarray. Science 270, 467-470.

Stimpson, D.I., Knepper, S.M., Shida, M., Obata, K. \& Tajima, H. (2004). Three-dimensional microarray platform applied to single nucleotide polymorphism analysis. Biotechnol Bioeng 87, 99-103.

Taylor, J.D., Briley, D., Nguyen, Q., Long, K., Iannone, M.A., Li, M.S., Ye, F., Afshari, A., Lai, E., Wagner, M., Chen, J. \& Weiner, M.P. (2001). Flow cytometric platform for highthroughput single nucleotide polymorphism analysis. Biotechniques 30, 661-666, 668-669.

Thaitrong N, Toriello NM, Del Bueno N, Mathies RA. (2009). Polymerase chain reactioncapillary electrophoresis genetic analysis microdevice with in-line affinity capture sample injection. Anal Chem. 81, 1371-7

Tojo, Y., Asahina, J., Miyashita, Y., Takahashi, M., Matsumoto, N., Hasegawa, S., Yohda, M. \& Tajima, H. (2005). Development of an automation system for single nucleotide polymorphisms genotyping using bio-strand, a new three-dimensional microarray. J Biosci Bioeng 99, 120-124 .

Wang, D.G., Fan, J.B., Siao, C.J., Berno, A., \& Young, P. et al. (1998). Large-scale identification, mapping, and genotyping of single-nucleotide polymorphisms in the human genome. Science 280, 1077-1082.

Wang, W., Kham, S.K., Yeo, G.H., Quah, T.C. \& Chong, S.S. (2003). Multiplex minisequencing screen for common Southeast Asian and Indian beta-thalassemia mutations. Clin Chem. 49, 209-218.

Xu, H., Sha, M.Y., Wong, E.Y., Uphoff, J., Xu, Y., Treadway, J.A., Truong, A., O'Brien, E., Asquith, S., Stubbins, M., Spurr, N.K., Lai, E.H. \& Mahoney, W. (2003). Multiplexed SNP genotyping using the Qbead system: a quantum dot-encoded microspherebased assay. Nucleic Acids Res 31, e43. 


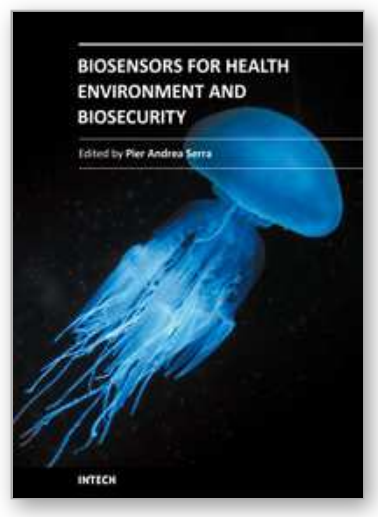

\author{
Biosensors for Health, Environment and Biosecurity \\ Edited by Prof. Pier Andrea Serra
}

ISBN 978-953-307-443-6

Hard cover, 540 pages

Publisher InTech

Published online 19, July, 2011

Published in print edition July, 2011

\begin{abstract}
A biosensor is a detecting device that combines a transducer with a biologically sensitive and selective component. Biosensors can measure compounds present in the environment, chemical processes, food and human body at low cost if compared with traditional analytical techniques. This book covers a wide range of aspects and issues related to biosensor technology, bringing together researchers from 16 different countries. The book consists of 24 chapters written by 76 authors and divided in three sections: Biosensors Technology and Materials, Biosensors for Health and Biosensors for Environment and Biosecurity.
\end{abstract}

\title{
How to reference
}

In order to correctly reference this scholarly work, feel free to copy and paste the following:

Johnson Kk Ng and Samuel Chong (2011). Multiplexing Capabilities of Biosensors for Clinical Diagnostics, Biosensors for Health, Environment and Biosecurity, Prof. Pier Andrea Serra (Ed.), ISBN: 978-953-307-443-6, InTech, Available from: http://www.intechopen.com/books/biosensors-for-health-environment-andbiosecurity/multiplexing-capabilities-of-biosensors-for-clinical-diagnostics

\section{INTECH}

open science | open minds

\section{InTech Europe}

University Campus STeP Ri

Slavka Krautzeka 83/A

51000 Rijeka, Croatia

Phone: +385 (51) 770447

Fax: +385 (51) 686166

www.intechopen.com

\section{InTech China}

Unit 405, Office Block, Hotel Equatorial Shanghai

No.65, Yan An Road (West), Shanghai, 200040, China

中国上海市延安西路65号上海国际贵都大饭店办公楼 405 单元

Phone: +86-21-62489820

Fax: +86-21-62489821 
(C) 2011 The Author(s). Licensee IntechOpen. This chapter is distributed under the terms of the Creative Commons Attribution-NonCommercialShareAlike-3.0 License, which permits use, distribution and reproduction for non-commercial purposes, provided the original is properly cited and derivative works building on this content are distributed under the same license. 